\title{
Michael SiLAGi
}

\section{Von Deutsch-Südwest zu Namibia, Wesen und Wandlungen des völkerrechtlichen Mandats.}

Verlag Rolf Gremer, Ebelsbach, Bd. 29 der Abhandlungen zur Rechtswissenschaftlichen Grundlagenforschung in der Reihe der Münchener Universitätsschriften (Juristische Fakultät), 165 S., 42,- DM

Die drei im südlichen Afrika schwelenden Brandherde - in und um die Republik Südafrika (RSA), in und um Südwestafrika/Namibia (SWA/N) und in und um Rhodesien/Zimbabwe $(\mathrm{Rh} / \mathrm{Z})$ - stehen zwar in engem räumlichen (,,geopolitischen“) und sonstigem Zusammenhange, weisen aber untereinander erhebliche Verschiedenheiten auf. Bei der RSA geht es um die Zukunft eines Raumes von 1224 Millionen qkm mit einer Bevölkerung von annähernd 27 Millionen; SWA/N umfaßt 823000 qkm, weist aber z. Zt. nur eine Bevölkerung von einer Million auf; Rh/Z umfaßt 390000 qkm, mit einer Bevölkerung von heute wohl rd. 3 Millionen. Bereits dieser Vergleich läßt erkennen, daß das überragende Schwergewicht aller Südafrikafragen in der RSA liegt und daß demgegenüber $\mathrm{SWA} / \mathrm{N}$ und $\mathrm{Rh} / \mathrm{Z}$ nur als verhältnismäßige kleine Randgebiete erscheinen. In der RSA macht die weiße Bevölkerung immerhin fast ein Fünftel (rd. 5 Millionen) der Gesamtbevölkerung des Landes aus, die aus Buren, Engländern, Franzosen und Deutschen zusammengeschmolzen, heute fraglos eine einheitliche „Nation“ bildet.

Demgegenüber zerfallen die nicht-weißen Bewohner der RSA in etwa 12 unterschiedliche Volksgruppen, die weder durch eine gemeinsame Sprache noch durch eine gemeinsame Kultur verbunden sind, die sich aber verständlicherweise dagegen wenden, daß ihnen ,Ethnizität" als Hindernis bei der Durchsetzung ihrer Rechte entgegengehalten wird. In SWA/N macht der Anteil der weißen Bevölkerung mit rd. 100000 nur etwa ein Zehntel der Gesamtbevölkerung aus; anders als in der RSA ist dieser Volksteil (im wesentlichen Buren und Deutsche) nach wie vor ein nach außen orientiertes Kolonialvolk geblieben. Die schwarze Bevölkerung des Landes setzt sich aus etwa 10 verschiedenen Volksgruppen mit erheblichen Unterschieden zusammen, wobei aber der Volksgruppe der Ovambos mit rd. 500000 Angehörigen hier ein deutliches Ubergewicht zukommt, das ihr politisch eine gewisse Führungsrolle gibt und demgegenüber auch hier eine Uberbetonung der ,Ethinizität“ nicht hingenommen wird. Wieder anders liegen die Bevölkerungsverhältnisse in Rh/Z. Das Mißverhältnis zwischen den bisher allein regierenden Weißen zur Mehrheit der Schwarzen ist hier mit $1: 20$ besonders kraß. Die hauchdünne weiße Bevölkerung besteht fast ausschließlich aus Engländern, die sich trotz des äußerlichen Bruchs mit dem Vereinigten Königreich auch weiterhin als solche und nur als solche fühlen und die im Hinblick auf den unabweisbaren Wandel der Dinge in ihre englische Heimat zurückzukehren sich anschicken. Die schwarze Bevölkerung besteht aus den beiden Stämmen der Matabele und Mashona, so daß sich hier Fragen einer mehr oder weniger ausgeprägten ,,Ethnizität“ kaum stellen. Aus alledem dürften sich Unterschiede im zeitlichen Ablauf ergeben. Der in jedem Falle zu erwartende Wandel der Dinge wird sich in und um die RSA sicherlich nur verhältnismäßig langfristig vollziehen können. Die Umgestaltung der Verhältnisse in SWA/N und $\mathrm{Rh} / \mathrm{Z}$ stellt sich dagegen immer deutlicher als eine dringende Gegenwartsfrage heraus. In greifbare Nähe ist dabei eine Lösung der SWA/Namibia-Frage gerückt, wobei im Augenblick freilich noch nicht absehbar ist, ob eine auch international, insbesondere von der Mehrheit der Vereinten Nationen anerkannte Lösung noch erhofft werden kann oder ob die RSA, die noch immer die tatsächliche Verfügungsgewalt über SWA/N ausübt, ohne weitere Rücksichtnahmen einen einseitigen Weg zur Gewährung der Unabhängigkeit für SWA/N gehen will. Fast täglich wechselt 
das Bild der Lage. Der besonderen Anteilnahme kann die Namibia-Frage in der Bundesrepublik insofern gewiß sein, als es sich hier um ein früheres deutsches Schutzgebiet handelt, in dem noch rd. 25000 Deutsche wohnen - sei es als förmliche deutsche Staatsbürger, deren Zahl etwa mit 6000 anzunehmen ist, sei es als sich jedenfalls sprachlich und kulturell noch zum Deutschtum zählende Südafrikaner. Eine weitere Besonderheit der SWA/N-Frage liegt darin, daß hier aus der Völkerbundszeit her noch das Mandatssystem eine gewisse Rolle spielt, mit der man seinerzeit den berühmten Punkt 5 der Wilson'schen 14 Punkte (,,Friede ohne alle Annexionen") zwar förmlich aufrecht halten zu können meinte, gleichwohl aber vor allem die C-Mandate der vollen tatsächlichen Souveränität der Mandatare unterstellte. Vor diesem Hintergrund und in diesem Rahmen ist auch das von Michael Silagi Ende 1978 vorgelegte Werk zu beurteilen, dessen Zeitnähe in die Augen springt und das damit einen wertvollen Beitrag zu den innerhalb und außerhalb der Vereinten Nationen über diese Frage stattfindenden Auseinandersetzungen liefert. In 3 Kapiteln behandelt der Verf. die ideengeschichtlichen Grundlagen des Südwestafrika-Mandates, den völkerrechtlichen Status Südwestafrikas und die Frage der Zuständigkeit des Internationalen Gerichtshofs in Den Haag zur Streitentscheidung in dieser Angelegenheit. In sorgfältigen und umfassenden Untersuchungen kommt Verf. zu dem Ergebnis, daß bereits mit dem Inkrafttreten des Friedensvertrages von Versailles (10. 1. 1920) die damalige Union von Südafrika (heute RSA) de jure Souverän des ehemaligen deutschen Schutzgebietes geworden ist, während das fast ein Jahr später (17. 12. 1920) vom Völkerbundsrat erlassene Mandatsstatut von sieben Artikeln dem Mandatar (Union of South Africa) keine weiteren Souveränitätsrechte mehr übertragen konnte und wollte, vielmehr von der bereits erworbenen Souveränität über das fragliche Gebiet ausgegangen ist und dazu nunmehr nur noch einige Verpflichtungen hinzufügte, die aber nach Art und Umfang nichts an der Tatsache der - fortdauernden - Souveränität der südafrikanischen Union hinsichtlich des Mandatsgebietes geändert haben. Besonders wichtig ist hierzu die Darlegung des Verf. , daß es bei näherer Prüfung der Mandatsakte vom 17. 2. 1920 a n jeder Symmetrie, jedem Gleichgewicht zwischen Rechten und Pflichten des Mandatars gebricht. Es nimmt nach alledem nicht wunder, daß der Verf. im dritten Kapitel seines Werkes zu dem Ergebnis kommt, daß der IGH in Den Haag keine Zuständigkeit zur Streitentscheidung über die Namibia-Frage hat, auch nicht über Art. 37 seines Statutes, und daß die gegenteilige Auffassung des IGH notwendig in eine Sackgasse führen muß, nichts anderes als Fehlanwendung des Völkerrechts (,,misapplication of international law“, S. 154) bedeutet. Um zu einer wirklich gerechten und tragfähigen Lösung der SWA/N-Frage zu gelangen, müsse man - unter Verzicht auf legalistische Sophistereien in Anknüpfung an das Mandatssystem des aufgelösten Völkerbundes - auf die moralischen und politischen Grundfragen der heutigen Entwicklung abstellen.

Obwohl die Ausführungen des Verf. der herrschenden Ansicht über die SWA/N-Frage weitgehend widersprechen, in Einzelheiten vielleicht auch nicht ganz überzeugend und jedenfalls nicht ohne gewisse Schwierigkeiten nachzuvollziehen sind - gelegentlich erscheinen die Gedankengänge des Verf. ein wenig zu scharfsinnig, als daß sie sofort eingängig sein könnten -, sowohl in seinen Ergebnissen als auch in seinen Beweisführungen wird man dem Verf. im wesentlichen nur zustimmen können. Die zuständigen Stellen der Vereinten Nationen und insbesondere auch die 5 mit der SWA/N-Frage besonders befaßten sog. Kontaktmächte (U. S. A., England, Canada, Frankreich und die Bundesrepublik) sollten sich in der Tat fragen, ob es nicht angezeigt ist, auf einen neuen, mehr moralisch-politischen Weg umzuschwenken, um so mehr, als sich hier die RSA mit ihrem beweglichen Außenminister Pick Botha durchaus verhandlungsbereit zeigt und schon einige ermutigende Vorleistungen er- 
bracht hat, wie z. B. die in SWA/N veranlaßte Aufhebung zahlreicher Apartheids-Vorschriften.

Der Wert und die Brauchbarkeit des besprochenen Werkes soll nicht eingeschränkt werden, wenn im Folgenden auch einige kritische Bemerkungen und Berichtigungen angebracht seien. Schon der Ausgangspunkt des Verf. auf S. 1 (, ,Schutzgebiet“ nur als Gebiet des Schutzes für missionierende, kolonisierende und handeltreibende deutsche Reichsangehörige zu verstehen, nicht aber als Gebiete des Schutzes für die Eingeborenen, nach Art des fragwürdigen Protektorats, dem im Jahre 1939 das Tschecho-Slovakische Volk unterworfen wurde) bedarf der Ergänzung. Nicht nur der Schutz deutscher Siedler, Missionare und Handelshäuser war der Sinn der Unterstellung SW A's unter die deutsche Schutzherrschaft; zahlreiche Verträge mit den Häuptlingen der verschiedenen Eingeborenenstämme des Landes, zumeist mit dem Erwerb weiter Ländereien für lächerlich geringe Preise verbunden, waren auch als ,,Schutzund Freundschaftsverträge" zum Besten der Eingeborenen geschlossen. Einzelheiten darüber sind auf S. 12 ff. des Werkes von Dr. W. Külz (Vater des Rezensenten) ,,Deutsch-Südafrika im 25. Jahre Deutscher Schutzherrschaft“, 1909 im Verlage Süsserot, nachzulesen. Die Originale dieser Verträge werden im Staatsarchiv zu Windhoek noch heute aufbewahrt; viele von ihnen tragen die Unterschrift des damals zuständigen Reichskommissars Dr. Göring, des Vaters des nachmaligen ,,Reichsmarschalls“ . . . Darüber, wie es dann wirklich mit diesem den eingeborenen Stämmen vom Reich zugesagten Schutz bestellt gewesen ist, brauchen weitere Worte nicht verloren zu werden.

Auf S. 13 f. erwähnt der Verf. zutreffend die weitgehenden burischen Sympathien für Deutschland, die sich aus deutscher Unterstützung während der Burenkriege ergeben (,,Krüger-Depesche u. a. m.) und zu erheblichem Widerstand geführt hatten. Auch hierzu erscheint eine Ergänzung angezeigt. Die Sympathien Südafrikas für Deutschland leiten sich nicht nur aus deutscher Unterstützung in den Burenkriegen ab, sondern auch daraus, daß ein nicht unerheblicher Teil der südafrikanischen Nation deutscher Herkunft und sich dieser Herkunft auch bis heute mehr oder weniger bewußt geblieben ist. Schon um die Mitte des vorigen Jahrhunderts wanderten auch Deutsche in Südafrika ein, die sich dann ständig vermehrten. Deutsche Namen finden wir denn auch bei den Inhabern bedeutender Staatsämter und vielfach im kulturellen und wirtschaftlichen Leben Südafrikas. Deutscher Herkunft waren z. B. der letzte Staatspräsident der RSA Dr. Diederichs, der langjährige Innen- und Justizminister des Landes Pirow (noch in Deutschland geboren); im kulturellen Bereich wäre etwa die aus einer deutschen Missionarsfamilie stammenden Schreiners zu nennen, aus der die bedeutende Dichterin Olive Schr. und einer der hervorragendsten Richter des Landes hervorgegangen sind. Auch der bekannte südafrikanische Industrielle Rupert stammt von deutschen Vorfahren ab. Südafrikaner deutscher Herkunft wurden vielfach gerade in SWA eingesetzt, wie z. B. einer der ersten Administratoren Hofmeyer und der erste burische Oberrichter des Landes Gutsche.

Auf S. 56 muß der Behauptung, daß ,,Südafrika in dem besetzten Deutsch-Südwest nach der deutschen Ubergabe sofort mit einer konsequenten Afrikanisierung begann", entschieden widersprochen werden, wenn damit mehr als die verwaltungsmäßige Einbeziehung des Landes in die Ordnungen Südafrikas gemeint sein sollte, wie etwa die Einbeziehung des Bahn- und Postsystems u. dgl. Bevölkerungsmäßig, wirtschaftlich und auch kulturell ist demgegenüber das Deutschtum in SWA nie ernstlich behelligt oder gar unterdrückt und ausgeschaltet worden. Die deutschen Farmer und Kaufleute konnten ohne alle Beschränkungen oder Zurücksetzungen wieder ihrer friedensmäßigen Beschäftigung nachgehen, die freien Berufe (Ärzte, Apotheker und Anwälte) ebenso, ohne daß sie etwa eine zusätzliche Prüfung hätten ablegen müssen. Die deutschen Schulen blieben unangetastet; deutsch wurde sogar als 
inoffizielle dritte Amtssprache eingeführt, in der alle Gesetze, Verordnungen u. dgl. neben Afrikaans und Englisch veröffentlicht wurden. Ebenso die verschiedenen deutschen Kirchen und Missionsanstalten. Erstaunlicherweise wurden die Deutschen in SWA auch während des Zweiten Weltkrieges nicht etwa kurzerhand enteignet, des Landes verwiesen oder in Lagern untergebracht. Letzteres wurde nur gegen einen Teil der männlichen deutschen Bevölkerung aus besonderen Gründen veranlaßt. Nach dem Ende des Krieges kehrten auch sie fast alle auf ihre Farmen und zu ihren sonstigen Berufen zurück; allein die Zahl der heute noch oder wieder in SWA/N lebenden Deutschen - 25000 - widerlegt die Annahme einer rücksichtslosen Afrikanisierungspolitik der Mandatsmacht. Die Zahl der in SWA/N lebenden Deutschen ist jedenfalls heute um ein Vielfaches höher, als je im kaiserlichen Schutzgebiet SWS gelebt haben.

Andererseits hatte die RSA nicht immer und für alle Zeiten dem Gedanken entsagt, SWA/N in ihr Staatsgebiet als fünfte Provinz einzugliedern. Der Mandatsstatus als solcher hätte dem nicht entgegengestanden. Den deutlichsten Schritt in diese Richtung hat die RSA mit ihrem 1940 versuchten Antrag auf völlige Einverleibung bei den Vereinten Nationen unternommen. Als der Antrag, wie zu erwarten, zurückgewiesen wurde, erließ die RSA im Jahre 1950 den South West Africa Affairs Amendment Act, auf Grund dessen das Land nunmehr in das südafrikanische Parlament 17 Abgeordnete zu wählen hatte und auch im Senat der RSA vertreten war; zu den in den Senat berufenen Volksvertretern gehörten u. a. auch zwei hervorragende deutsche Vertreter, der Sprach- und Kulturforscher Vedder und nach ihm der langjährige Rektor der deutschen Regierungsschule in Windhoek, Frei. Erst um die Wende der sechziger zu den siebziger Jahren schlug die RSA einen anderen Kurs ein, den auf Selbständigkeit abzielenden, allgemein als Turnhallen-Kurs bezeichneten Kurs, der im Jahre 1975 zum Zusammentritt der sog. Turnhallen-Versammlung, die - aus Vertretern aller Völkerschaften unter dem englischen Vorsitzenden Dirk Mudge zusammengesetzt - den Entwurf einer Verfassung für das Land als selbständiger, unabhängiger Staat auszuarbeiten hatte, welchen Auftrag die Turnhallenversammlung auch erfüllte. Mit diesem Markstein in der Geschichte des Landes entfielen natürlich auch die bisher in die südafrikanischen Gesetzgebungskörperschaften zu berufenden Abgeordneten und Senatoren. Bis in diese Tage hinein wird die SWA/N-Szene die Turnhallen-Verfassung beherrscht haben, die aber nach anfänglicher anderer Einstellung der RSA auch noch nicht das letzte Wort in der Verfassungsgebung für das neue SWA/N sein soll. Es verwundert ein wenig, daß das hier besprochene Werk mit keinem Wort auf diese Entwicklungen einzugehen für nötig befunden hat. Die SWA Affairs Amendment Act von 1950 und der von der Turnhalle ausgearbeitete Verfassungsentwurf von 1977 hätten zumindest kurz erwähnt werden sollen, was aber, wie zum Schluß wiederholt sei, den Wert der Arbeit nicht in Frage stellt.

Helmut R. Külz

Coulson, Michael C.

Arab Politics - The Search For Legitimacy

Yale University Press, New Haven-London 1977

Der Verfasser analysiert die politische Situation in den Staaten der arabischen Welt. Sein Buch ist in zwei annähernd gleich große Teile gegliedert, deren erster die Darstellung der drei Hauptmodelle für sozialen Wandel umfaßt - also mehr theoretisch orientiert ist -, deren zweiter die praktische Bewältigung des Legitimitätsproblems an Beispielsfällen einzelner arabischer Staaten darstellt. Bedauerlicherweise ist nicht ersichtlich, nach welchen Kriterien die Auswahl getroffen wurde, warum z. B. Pakistan und der Iran nicht angesprochen wer- 